\title{
La enseñanza del entorno a través de rincones
}

\author{
MARÍA PUIG GUTIÉRREZ \\ Universidad de Sevilla \\ Dpto. Didáctica de las Ciencias \\ Experimentales y Sociales \\ mpuig@us.es \\ ORCID: https://orcid.org/0000-0002-7536-2976 \\ D.O.I.: http://dx.doi.org/10.12795/JDU.2018.i01.104 \\ Pp.: 1854-1872
}

\section{Resumen}

La presente comunicación recoge la experiencia realizada con alumnos/as del tercer curso del Grado de Educación Infantil, en el marco de la asignatura Conocimiento del Entorno Social, al tratar de llevar a cabo un Ciclo de Mejora Docente (CMD). Dicho ciclo tiene como peculiaridad que se desarrolla de forma coordinada con la asignatura de Enseñanza del Entorno Natural, por lo que se trata de una experiencia de coordinación docente. Centrado en el diseño de rincones, buscamos el aprendizaje activo por parte del alumnado y la conexión con la práctica.

Palabras claves: Conocimiento del entorno social, educación infantil, docencia universitaria, experimentación docente universitaria, coordinación docente. 


\section{Breve descripción del contexto}

Durante este curso el ciclo de mejora docente que hemos llevado a cabo, vuelve a estar orientado no solo al diseño de una unidad o bloque de contenidos de la asignatura de Conocimiento del Entorno Social de 3 o del Grado de Educación Infantil, sino que en línea con anteriores participaciones en la red, seguimos apostando por la coordinación docente. Así, trabajamos de forma coordinada con la profesora de la asignatura del grupo 1 de Enseñanza del Entorno Natural. Ambas asignaturas fueron diseñadas en sus orígenes como complementarias, y desde hace unos años venimos trabajando la coordinación, incorporando cada vez más bloques de contenidos que se organizan de forma conjunta. De este modo, seguimos apostando por una coordinación real que nos permita trabajar coordinadamente ambas materias con el alumnado, lo que implica reformular el bloque de contenido o unidad, diseñar las sesiones de trabajo contando con las horas de ambas materias y reunirnos en muchas ocasiones para que de verdad el alumnado perciba que están trabajando con docentes diferentes, pero con un mismo objetivo de aprendizaje.

En lo que respecta al grupo de alumnos, está compuesto por un total de 75 personas, de los cuales 65 son los que suelen acudir con regularidad y están matriculados en las dos asignaturas implicadas. De estos 65, cuatro son hombres y el resto mujeres. La asignatura de Conocimiento del Entorno Social, es una materia obligatoria de 6 créditos, que vengo impartiendo desde el curso 2012-13. Se trata de una materia práctica que suele resultar atractiva al alumnado y que cursan con dedicación pero sin demasiadas dificultades. Como es habitual, la mayoría de los alumnos matriculados ha optado por la modalidad presencial, para la que es necesario la asistencia al $80 \%$ de las clases.

Por lo general, los alumnos/as se muestran implicados en el desarrollo de las distintas tareas, suelen participar de forma activa en los debates y parecen entusiasmados por su carrera y por la asignatura. Suele ocurrir casi siempre que hay algunos que se ven más comprometidos que 
otros, siendo los que más participan en clase y más dudas se plantean. Finalmente debemos añadir, que contamos con aulas en las que el mobiliario facilita el trabajo en equipo, puesto que están especialmente diseñadas para ello y es esta la forma habitual de trabajo en clase.

\section{Diseño previo del Ciclo de Mejora Docente}

Describimos a continuación los diferentes elementos que componían el diseño previo del ciclo de mejora:

\section{Mapa de contenidos}

El mapa de problemas que diseñamos se estructura en torno a un problema central “ ¿Son los rincones una buena estrategia metodológica para la Enseñanza del Entorno en Educación Infantil?". De esta pregunta, derivan tres subproblemas que nos permiten ir abordando los diferentes contenidos que hemos considerado esenciales para este bloque. Como se puede apreciar, de alguno de estos subproblemas derivan otros que creemos de segundo nivel y que aparecen con una letra algo más pequeña y con un color más claro. A través de flechas hemos señalado las relaciones que existen entre los contenidos. Así, por ejemplo, hemos empleado flechas de color rojo para marcar la relación entre las cuestiones claves y flechas bidireccionales para señalar la relación bidireccional entre algunos de los contenidos recogidos. Hemos usado también un código de color para diferenciar los contenidos de carácter conceptual (rojo), de los que tienen carácter procedimental (verde) y de los de carácter actitudinal, que hemos considerado transversales para todos los bloques y hemos colocado en tres pequeños círculos morados dispersos por el mapa. 


\begin{tabular}{|c|c|c|}
\hline $\begin{array}{l}\text { - Conceptualización } \\
\text { - Fundamentos teóricos } \\
\text { - Principales características }\end{array}$ & $\begin{array}{l}\text { - Organización del aula } \\
\text { - Organización del } \\
\text { alumnado } \\
\text { - Planificación del rincón } \\
\text { (ambientación) }\end{array}$ & $\begin{array}{l}\text { - Rincones convencionales } \\
\text { - Rincones temáticos } \\
\text { - Análisis de los tipos de } \\
\text { rincones }\end{array}$ \\
\hline
\end{tabular}

- Posibilidades del área de Conocimiento del Entorno como núcleo central

Interpretación y análisis del curriculum oficial
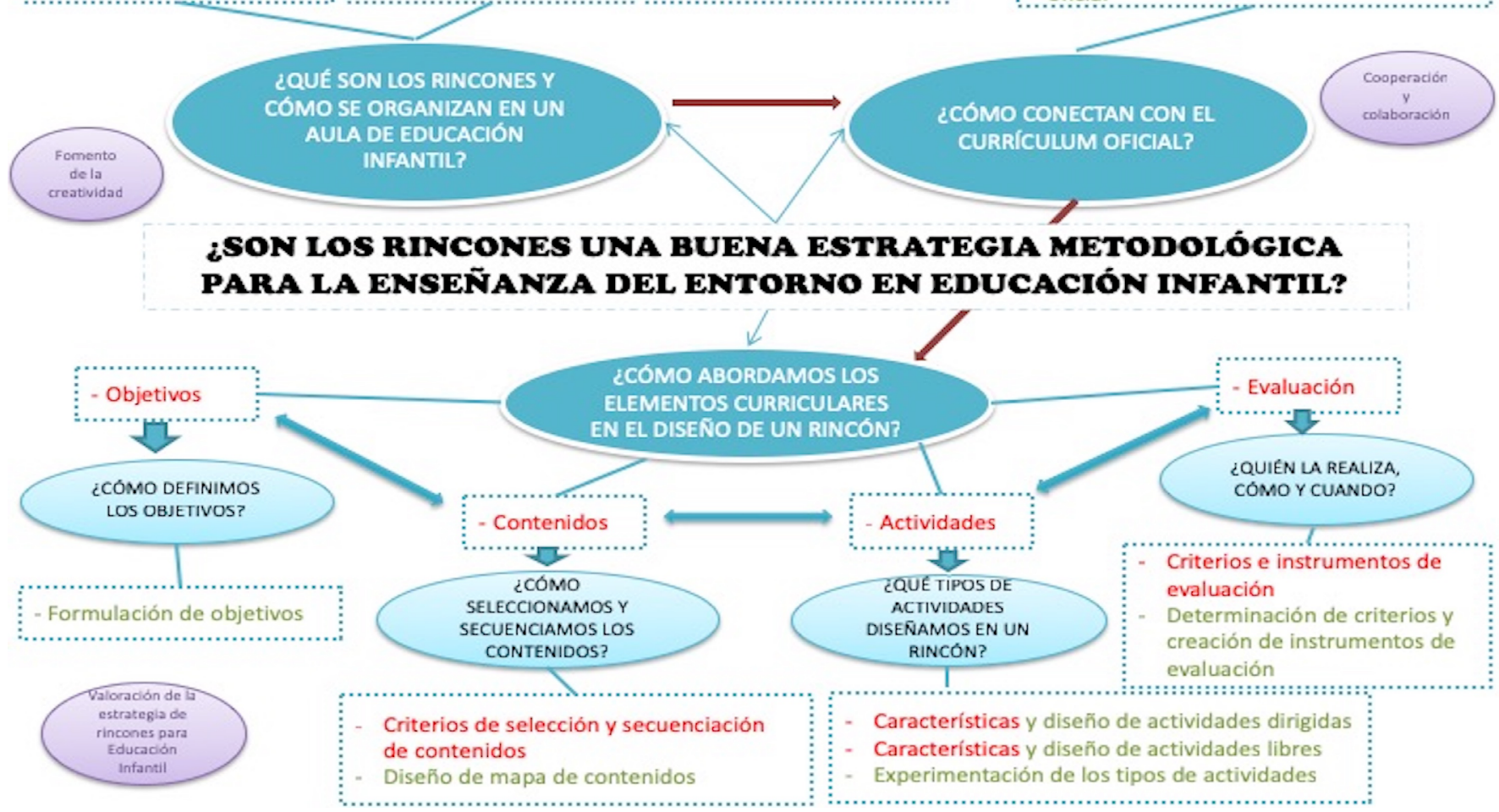

Figura 1. Mapa de problemas

Jornadas de Formación e Innovación Docente del Profesorado | № 1 (2018)

(c) (i) $\odot$ Esta obra se distribuye con la licencia Creative Commons Internacional (CC BY-NC-ND 4.0.) 


\section{Modelo metodológico posible y secuencia de actividades programadas}

El modelo metodológico que hasta ahora ha guiado mis CMD se ha centrado en establecer una estrecha relación entre teoría y práctica, siempre tomando como protagonista al alumnado. Para lograr esto, se trabaja en torno a problemas relevantes, que hay que identificar, para posteriormente secuenciar y elaborar una trama que nos sirva como guía en todo el proceso de aprendizaje. En lo que respecta al diseño de actividades, determinamos algunas características que deben cumplir: estar vinculadas con la práctica profesional, no tener una solución única y correcta, precisar de la reflexión y la construcción conjunta del conocimiento, considerar las ideas iniciales del alumnado, así como la evolución de las mismas y dar respuesta a las problemáticas planteadas. En este modelo, las clases han sido consideradas lugares y momentos en los que compartir ideas y construir entre todos nuevas formas de hacer frente a la realidad profesional. Para ello creemos esencial la creación de un buen clima de aula, donde el diálogo y la reflexión son las herramientas principales. Así, en este modelo apostamos por comenzar acercándonos a las ideas del alumnado, introducir una actividad motivadora que capte su atención para iniciar el trabajo, ofrecer información nueva y favorecer el contraste entre esta y las ideas iniciales, para finalmente realizar tareas de recapitulación. En lo que respecta a la evaluación, los informes de trabajo han sido hasta ahora los instrumentos empleados, que han permitido dejar plasmadas las diferentes actividades, las reflexiones generadas y el aprendizaje logrado.

Jornadas de Formación e Innovación Docente del Profesorado I № 1 (2018) Esta obra se distribuye con la licencia Creative Commons 


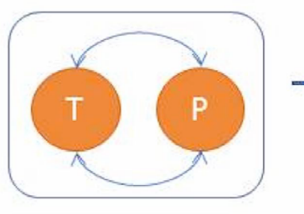

$$
\text { Determinación de }
$$
problemas relevantes

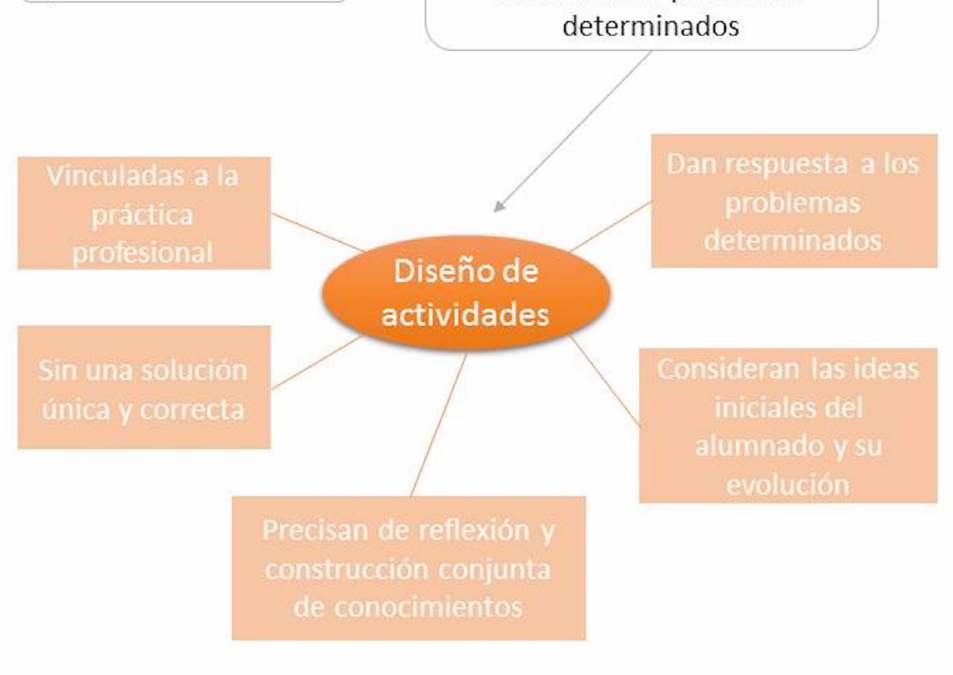

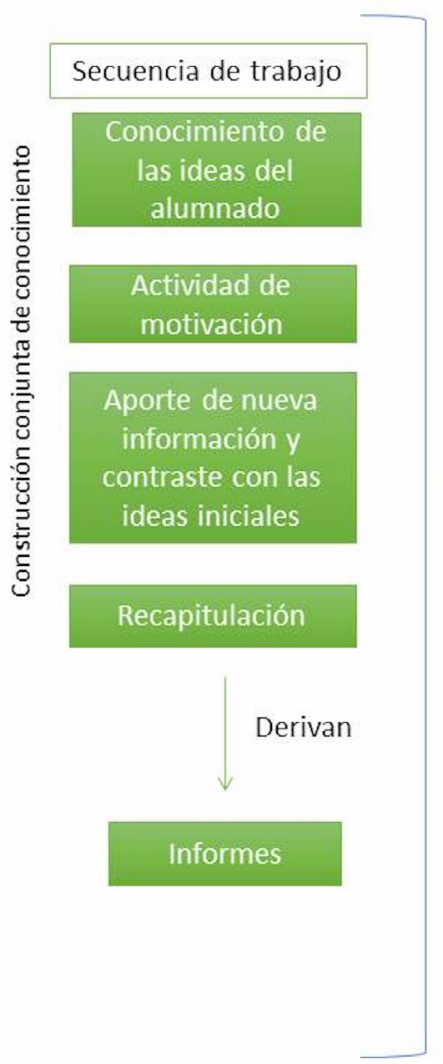

Figura 2. Modelo metodológico

Selección y secuenciación de contenidos en forma de trama

en base a los problemas determinados

Jornadas de Formación e Innovación Docente del Profesorado | № 1 (2018) cC (i) $\ominus$ Esta obra se distribuye con la licencia Creative Commons

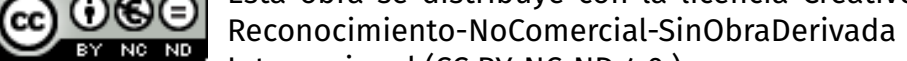
Internacional (CC BY-NC-ND 4.0.) 
A continuación, mostramos la secuencia de actividades prevista.

\section{Tabla 1}

\section{Secuencia de actividades prevista}

\begin{tabular}{|c|c|}
\hline IDEAS INICIALES & $\begin{array}{l}\text { Sesión } 1 \text { ¿Qué son los rincones y cómo se organizan en } \\
\text { un aula de Educación Infantil? } \\
\text { Presentación del bloque. } \\
\text { Organización de los grupos de trabajo. } \\
\text { Cuestionario de ideas iniciales. } \\
\text { Puesta en común de las respuestas ofrecidas en los } \\
\text { cuestionarios iniciales. }\end{array}$ \\
\hline $\begin{array}{l}\text { AMPLIACIÓN Y } \\
\text { CONTRASTRE }\end{array}$ & $\begin{array}{l}\text { Lectura de documentos seleccionado por las docentes } \\
\text { sobre los rincones en pequeño grupo extrayendo las } \\
\text { ideas principales. } \\
\text { Puesta en común entre los grupos que hayan tenido } \\
\text { la misma lectura elaborando síntesis común de ideas } \\
\text { principales. } \\
\text { Puesta en común en gran grupo donde cada lectura es } \\
\text { presentada con sus ideas principales. }\end{array}$ \\
\hline RECAPITULACIÓN & $\begin{array}{l}\text { Sintesis de ideas por parte de las docentes apoyada en } \\
\text { ppt. }\end{array}$ \\
\hline motivación & $\begin{array}{l}\text { Sesión } 2 \text { ¿Cómo conectan con el currículum oficial? } \\
\text { Vídeo introductorio sobre los tipos de rincones. } \\
\text { Presentación del informe }\end{array}$ \\
\hline $\begin{array}{l}\text { AMPLIACIÓN Y } \\
\text { CONTRASTRE }\end{array}$ & $\begin{array}{l}\text { Selección de temáticas del rincón por cada grupo, en } \\
\text { base a un listado dado por las docentes. } \\
\text { Justificación de la temática en base al currículum: } \\
\text { - } \quad \text { Breve presentación dialogada sobre el marco legal. } \\
\text { - } \quad \text { Lectura de la Orden que regula el currículum de } \\
\text { Ed. Infantil. } \\
\text { - } \quad \text { Elaboración y justificación del valor educativo de la } \\
\text { temática elegida en base al marco legal. } \\
\text { Objetivos: } \\
\text { - } \quad \text { Exposición dialogada sobre la formulación de } \\
\text { objetivos. } \\
\text { - } \quad \text { Definición de los objetivos de cada rincón. }\end{array}$ \\
\hline Recapitı & $\begin{array}{l}\text { Puesta en común en gran grupo lo trabajado en la } \\
\text { sesión. }\end{array}$ \\
\hline
\end{tabular}

Jornadas de Formación e Innovación Docente del Profesorado | № 1 (2018) Esta obra se distribuye con la licencia Creative Commons 


\begin{tabular}{|c|c|}
\hline motivación & $\begin{array}{l}\text { Sesión } 3 \text { ¿Cómo seleccionamos y secuenciamos los } \\
\text { contenidos? } \\
\text { Presentación de recursos y materiales sobre algunas de } \\
\text { las temáticas elegidas }\end{array}$ \\
\hline $\begin{array}{l}\text { AMPLIACIÓN Y } \\
\text { CONTRASTE }\end{array}$ & $\begin{array}{l}\text { Elaboración de un dossier con información sobre el } \\
\text { contenido que aborda cada uno de los rincones. Cada } \\
\text { grupo debe informarse sobre la temática que va a tra- } \\
\text { bajar y preparar un dossier con documentación. }\end{array}$ \\
\hline RECAPITULACIÓN & $\begin{array}{l}\text { Sesión } 4 \\
\text { Puesta en común en gran grupo de los dossier } \\
\text { elaborados. }\end{array}$ \\
\hline \multirow{2}{*}{$\begin{array}{l}\text { AMPLIACIÓN Y } \\
\text { CONTRASTE }\end{array}$} & $\begin{array}{l}\text { Presentación de mapas de contenidos elaborados por } \\
\text { docentes y por niños. } \\
\text { Diálogo sobre las posibilidades de los mapas para la } \\
\text { selección y secuenciación del contenido. }\end{array}$ \\
\hline & $\begin{array}{l}\text { Sesión } 5 \\
\text { Elaboración de mapas de contenidos para cada uno de } \\
\text { los rincones }\end{array}$ \\
\hline RECAPITULACIÓN & $\begin{array}{l}\text { Puesta en común en gran grupo de los mapas } \\
\text { elaborados. }\end{array}$ \\
\hline MOTIVACIÓN & $\begin{array}{l}\text { Sesión } 6 \text { ¿Qué tipos de actividades diseñamos en un } \\
\text { rincón? } \\
\text { Realización de actividades libres y dirigidas planteadas } \\
\text { por las docentes }\end{array}$ \\
\hline $\begin{array}{l}\text { AMPLIACIÓN Y } \\
\text { CONTRASTE }\end{array}$ & $\begin{array}{l}\text { Exposición dialogada sobre los tipos de actividades } \\
\text { Diseño de actividades libres y dirigidas para cada } \\
\text { rincón }\end{array}$ \\
\hline RECAPITULACIÓN & Puesta en común de las actividades diseñadas \\
\hline MOTIVACIÓN & $\begin{array}{l}\text { Sesión } 7 \text { ¿Quién realiza la evaluación, cómo y cuándo? } \\
\text { Debate sobre la evaluación de los rincones }\end{array}$ \\
\hline $\begin{array}{l}\text { AMPLIACIÓN Y } \\
\text { CONTRASTE }\end{array}$ & $\begin{array}{l}\text { Exposición dialogada con apoyo de ejemplos prácticos } \\
\text { para la formulación de criterios de evaluación y el di- } \\
\text { seño de instrumentos. } \\
\text { Formulación de criterios de evaluación y diseño de al- } \\
\text { gún instrumento para cada rincón. }\end{array}$ \\
\hline RECAPITULACIÓN & $\begin{array}{l}\text { Puesta en común de los criterios e instrumentos } \\
\text { elaborados. }\end{array}$ \\
\hline $\begin{array}{l}\text { SÍNTESIS Y CO- } \\
\text { MUNCACIÓN DE LO } \\
\text { APRENDIDO }\end{array}$ & $\begin{array}{l}\text { Sesión } 8 \\
\text { Exposición de los rincones diseñados }\end{array}$ \\
\hline IDEAS FINALES & $\begin{array}{l}\text { Sesión } 9 \\
\text { Valoración global del bloque y recogida de ideas finales }\end{array}$ \\
\hline
\end{tabular}

Jornadas de Formación e Innovación Docente del Profesorado | № 1 (2018) Esta obra se distribuye con la licencia Creative Commons Reconocimiento-NoComercial-SinObraDerivada Internacional (CC BY-NC-ND 4.0.) 


\section{Cuestionario inicial-final para hacer seguimiento de la evolución de los estudiantes}

Para el seguimiento de la evolución del aprendizaje de los alumnos/as, diseñamos un cuestionario de 10 preguntas abiertas relacionadas con los tres grandes problemas que planteamos. Puesto que no pudimos llevar a cabo el bloque de contenidos completo por falta de tiempo, al final del CMD solo se planteó a los estudiantes las seis primeras cuestiones. Todas las preguntas formuladas eran abiertas, de forma que los alumnos respondían expresando sus ideas de manera libre.

\section{Aplicación del CMD}

\section{Relato resumido de las sesiones, describiendo y analizando el clima de trabajo, la actuación docente propia, el desarrollo real de las actividades, los avances y dificultades encontradas.}

Antes de comenzar conviene aclarar que la secuencia prevista no ha sido realizada en su conjunto en el tiempo establecido. La complejidad y amplitud de los contenidos abordados, junto a la dificultad para llevar en paralelo este bloque con otros de las asignaturas implicadas en el ciclo, han sido la causa fundamental de este desajuste.

El inicio de la secuencia transcurrió como estaba previsto, una vez presentado el bloque y organizados los grupos de trabajo, realizamos el cuestionario de ideas iniciales, con su posterior puesta en común. Los alumnos mostraron interés en profundizar más sobre esta metodología, ya que, aunque era conocida por muchos, surgieron multitud de interrogantes. Tras esta primera parte, repartimos las lecturas y llevamos a cabo la dinámica prevista. 
De este modo, fueron aclarándose algunos de los interrogantes antes planteados. La dinámica permitió que los alumnos realizaran varias lecturas de un mismo texto y que extrajeran las ideas principales, contrastando con las encontradas por otros compañeros. Resultó bastante enriquecedora, aunque se alargara en el tiempo. Tras la puesta en común de las lecturas, ofrecimos una síntesis de ideas esenciales sobre los rincones apoyándonos en una presentación.

La sesión dos también transcurrió como estaba previsto, de modo que tras un video introductorio, presentamos el informe que debían elaborar. Posteriormente ofrecimos a los alumnos un listado de 26 problemáticas, en las que tratábamos de conjugar temáticas más propias del área de Entorno Natural, con otras más cercanas al Entorno Social. Después de barajar muchas opciones, decidimos quedarnos con 26 problemas, formulados en forma de pregunta, para que así ya tuvieran al menos la pregunta inicial que desencadenara posteriormente su propuesta didáctica. Para evitar conflictos, decidimos meter en una bolsa todos los números de los grupos, y conforme iban saliendo, iban eligiendo. Dimos la opción de que hubiera dos grupos en un mismo problema y en general el proceso de elección fue tranquilo y casi todos los grupos escogieron la temática que quisieron.

Elegida la temática sobre la que diseñarían el rincón, nos acercamos a la legislación para tratar de justificar desde el marco legal el tema objeto de estudio. Como suele ocurrir, la lectura de la Orden no resulta demasiado atractiva, pero realmente fue bastante útil para justificar el valor educativo del problema elegido. A continuación, nos adentramos en la formulación de los objetivos, para lo que realizamos primero una exposición dialogada con las claves para formular objetivos y posteriormente cada grupo procedió a desarrollar los objetivos propios de su rincón. La sesión finalizó con la puesta en común de los objetivos planteados por cada grupo. Esta puesta en común resultó 
bastante rica, en cuanto se retomaron ideas trabajadas en la sesión anterior respecto a los rincones, así como se aclararon algunos errores comunes a la hora de formular objetivos.

En la siguiente sesión comenzamos presentando algunos libros, juegos y otros materiales sobre algunos de los temas elegidos. Esto llamó su atención y nos sirvió para conectar con la importancia de conocer el contenido de la propuesta didáctica que iban a diseñar. Así, planteamos la elaboración de un dossier en el que recogieran información sobre el problema en torno al que giraría el rincón. Tuvimos que aclarar en múltiples ocasiones que el objetivo del dossier era conocer en profundidad la temática y que, por lo tanto, no estaba dirigido a niños, sino a ellos y ellas como futuros docentes. La elaboración del dossier les llevó más tiempo del previsto, tomando parte de la siguiente sesión, en la que finalizamos con la puesta en común.

Para la sesión seis llevamos ejemplos de mapas de contenido, algunos elaborados por docentes y otros incluso por niños. Partiendo de lo que habían visto, fuimos comentando y planteamos la elaboración de mapas de contenidos para cada uno de los rincones. Así, tras ofrecer algunas pautas para ello, cada grupo dedicó toda la sesión a la elaboración de su mapa de problemas, en el que recoger todos los contenidos que pretendían abordar. Esta tarea resultó complicada puesto que, hasta el momento, siempre a la hora de seleccionar contenidos, lo habían hecho a modo de lista. Establecer relaciones entre los contenidos y formular preguntas cercanas al alumnado fueron los dos grandes obstáculos encontrados. Como un hecho curioso, podemos destacar que, a pesar de haberles dado una pregunta inicial, cuando elaboraron los mapas de contenidos, muchos grupos cambiaban la pregunta por un tema. Al final de la sesión comenzamos la puesta en común, pero esta se alargó hasta la mitad de la sesión siete, dado que se iba exponiendo cada mapa y se formulaban 
preguntas por parte de los compañeros y de las docentes. Llegados a este punto, fuimos conscientes de la necesidad de conectar lo que estábamos trabajando con las ideas de los alumnos, por lo que decidimos cruzar el análisis de las ideas de los niños previsto en la otra parte de las asignaturas con lo que habían realizado hasta el momento. Así, cada grupo debía preparar un instrumento para recoger las ideas de los alumnos sobre su problemática. Comenzamos pues, viendo un vídeo sobre las ideas de los alumnos, en el que se presentan sus principales características y se muestran diversas asambleas llevadas a cabo en las aulas de infantil, permitiendo acercar al alumnado a la realidad del aula. Tras ofrecer algunas pautas sobre como diseñar un instrumento para conocer las ideas de los alumnos, comenzamos el diseño. Los instrumentos debían tener claras las preguntas que se hacían y el objetivo que se perseguía con ella. Cuando todos los grupos habían diseñado su instrumento, fuimos a un centro para que pudieran aplicarlo con un grupo de alumnos. Esta salida resultó muy motivadora, aunque supuso la incorporación de tiempo extra en horario que no era la clase de los viernes, tal y como habíamos hecho hasta el momento. Tras la recogida de ideas, que fueron grabadas en audio, los estudiantes realizaron un análisis determinando perfiles y estableciendo niveles de complejidad del conocimiento de los niños y niñas, al tiempo que identificaban las características del pensamiento infantil. Es justo en este punto, en el que nos encontramos cuando estamos redactando esta comunicación, por lo que no hemos podido abordar los contenidos previstos relacionados con el diseño de actividades y la evaluación. Tampoco hemos llegado aún a la exposición de los rincones diseñados. Continuaremos trabajando para lograrlo antes del fin del cuatrimestre. 
Evaluación del aprendizaje de los estudiantes, comparando sus conocimientos iniciales y finales.

Para determinar el aprendizaje de los alumnos/as, se diseñó un instrumento que se aplicó de forma individual al inicio y al final de la unidad. Los datos obtenidos en ambos momentos nos permiten conocer la evolución de los aprendizajes. Una vez que los alumnos completaron el cuestionario, analizamos sus respuestas determinando niveles de complejidad, de forma que ordenamos las respuestas de las más simples a las más complejas y calculamos el porcentaje de alumnos que estaba en cada nivel. A continuación podemos ver las escaleras de aprendizaje, en las que es posible identificar cada uno de los niveles, así como el porcentaje de alumnos que se sitúa en ellos al inicio y al final del ciclo de mejora.

En la imagen de la primera escalera (Figura 3) podemos apreciar que las ideas de los alumnos sobre la metodología de los rincones evoluciona favorablemente tras el CMD. Si en el inicio ningún alumno llegaba a dar una respuesta del nivel 4, al final más del $80 \%$ se sitúa en él.

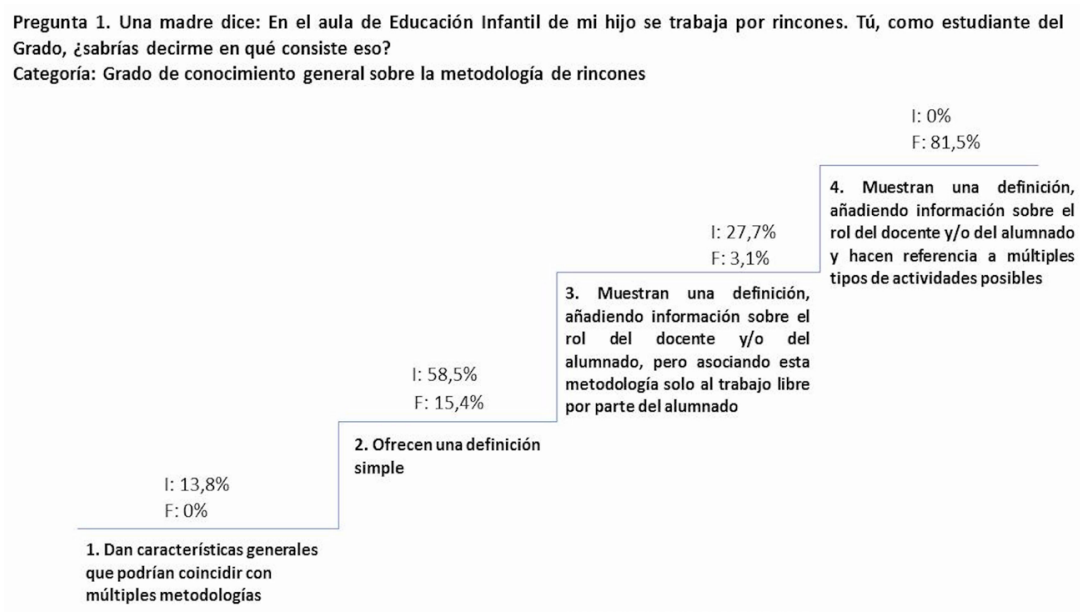

Figura 3. Escalera de aprendizaje Pregunta 1

Jornadas de Formación e Innovación Docente del Profesorado | № 1 (2018) Esta obra se distribuye con la licencia Creative Commons Reconocimiento-NoComercial-SinObraDerivada Internacional (CC BY-NC-ND 4.0.) 
En la pregunta dos también apreciamos evolución y podemos ver cómo al inicio los porcentajes más amplios de respuestas se encuentran en los niveles iniciales y al final se localizan en los más elevados. De este modo, el 73,9\% del alumnado está al final en el nivel 4 y ningún estudiante se encuentra en el nivel 1.

Pregunta 2. La otra madre te pregunta: ¿Me puedes decir entonces como se organiza la clase (espacio y alumnos) para llevar a cabo los rincones?

Categoría: Grado de conocimiento sobre la organización del aula atendiendo a la metodología de rincones

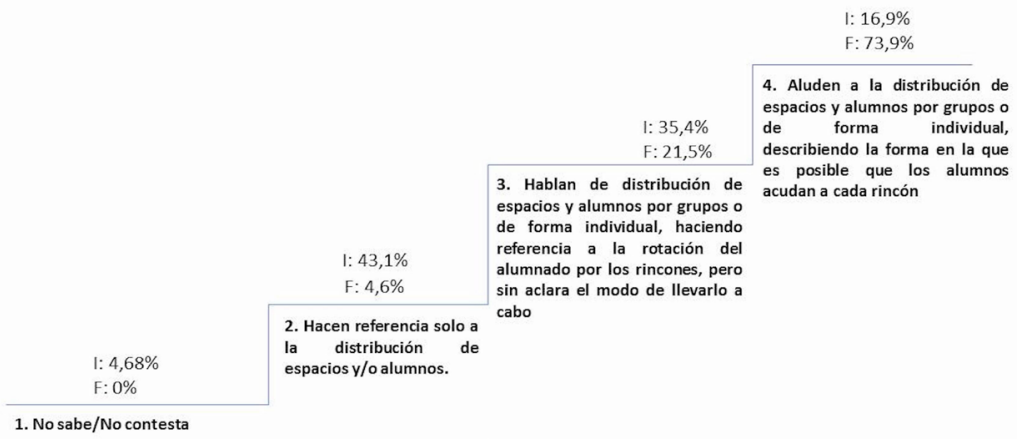

Figura 4. Escalera de aprendizaje Pregunta 2

Al preguntar por los tipos de rincones, se aprecia en la figura que tras el CMD, las ideas de los alumnos evolucionan hacia niveles más complejos. En este caso, la evolución es bastante llamativa, ya que al inicio más del 50\% del alumnado se situaba en el nivel 1 y al final el 67,7\% alcanza el nivel 4.

Pregunta 3. En este momento todavía un padre no se hace una idea de lo que le estas comentando y te pregunta: ¿Podrías ponerme algunos ejemplos para hacerme una idea?

Categoría: Grado de conocimiento sobre los tipos de rincones

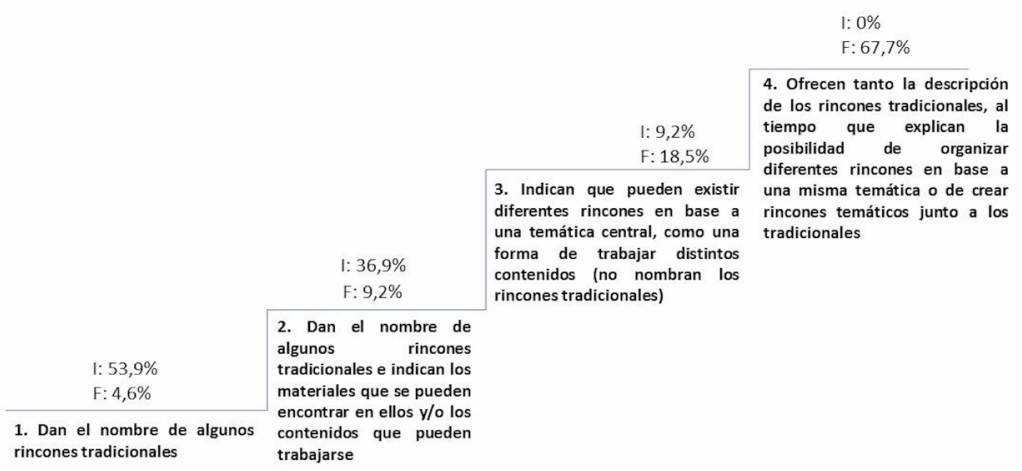

Figura 5. Escalera de aprendizaje Pregunta 3

Jornadas de Formación e Innovación Docente del Profesorado I № 1 (2018) Esta obra se distribuye con la licencia Creative Commons Reconocimiento-NoComercial-SinObraDerivada Internacional (CC BY-NC-ND 4.0.) 
En la pregunta cuatro vemos que en el momento inicial más del 50\% del alumnado hace alusión a temáticas relacionadas con entorno social o entorno natural, siendo predominantes los que señalan este último. Sin embargo, en el momento final, más del $80 \%$ de los estudiantes aluden a temáticas tanto de carácter social como natural. La tradición del uso de los rincones se basa fundamentalmente en actividades prácticas, que suelen estar más asociadas a temáticas de naturales (las plantas, los animales, la contaminación, los residuos), por lo que consideramos que esto es una de las razones de las respuestas iniciales.

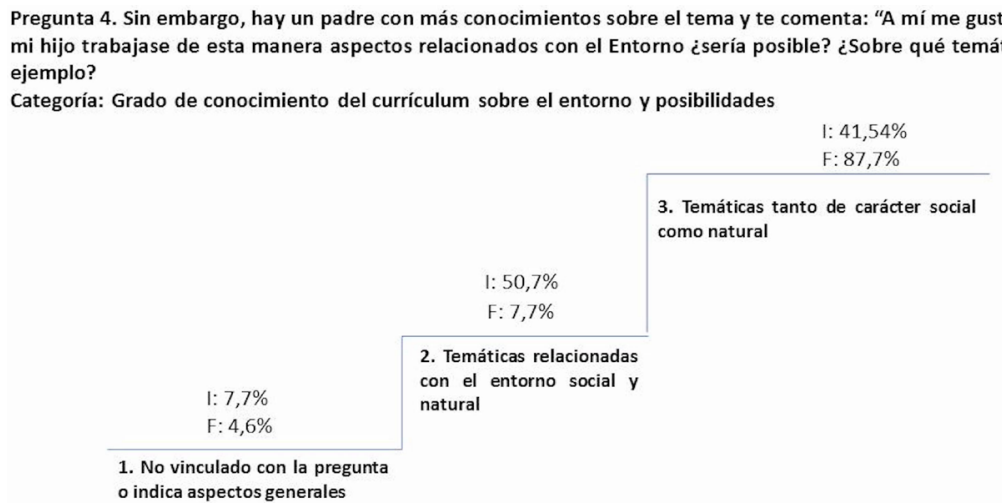

Figura 6. Escalera de aprendizaje Pregunta 4

En cuanto a los objetivos, nos damos cuenta como en el momento inicial un porcentaje próximo al 50\% plantea objetivos generales, sin vinculación con los contenidos específicos de los rincones. Sin embargo, en el momento final los alumnos no solo plantean objetivos generales, sino que son capaces de formular objetivos específicos de los diferentes rincones.

Jornadas de Formación e Innovación Docente del Profesorado | № 1 (2018) Esta obra se distribuye con la licencia Creative Commons Reconocimiento-NoComercial-SinObraDerivada Internacional (CC BY-NC-ND 4.0.) 
Pregunta 5. ¿Se plantean objetivos a la hora de diseñar un rincón? Pon algún ejemplo Categoría: Grado de conocimiento sobre el elemento curricular objetivos

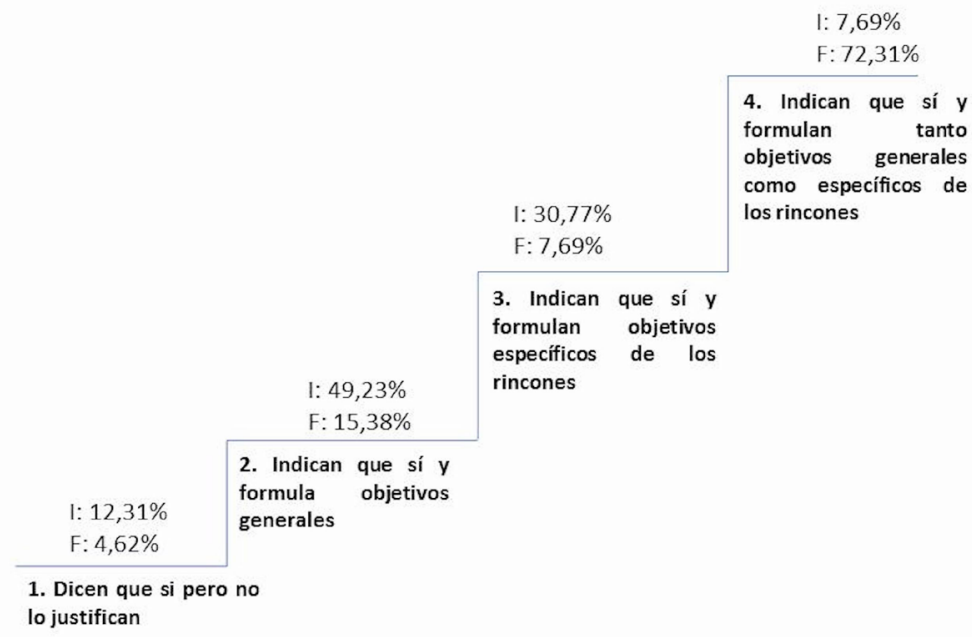

Figura 7. Escalera de aprendizaje Pregunta 5

En la primera parte de la pregunta 6 , en el momento inicial existe un porcentaje muy alto de respuestas que se posicionan en el valor 1, y consideramos que es debido a la redacción poco clara de la pregunta. Por este motivo, en el momento final se reformuló la pregunta indicando ¿qué papel tienen los contenidos? ¿de qué tipo son los contenidos que se pretenden enseñar? Al realizar este cambio, en el momento final, encontramos respuestas que se posicionan fundamentalmente en el valor 2 y 3.

Pregunta 6 (primera parte). ¿Qué papel tienen los contenidos?

Categoría: Grado de conocimiento del contenido curricular: contenidos

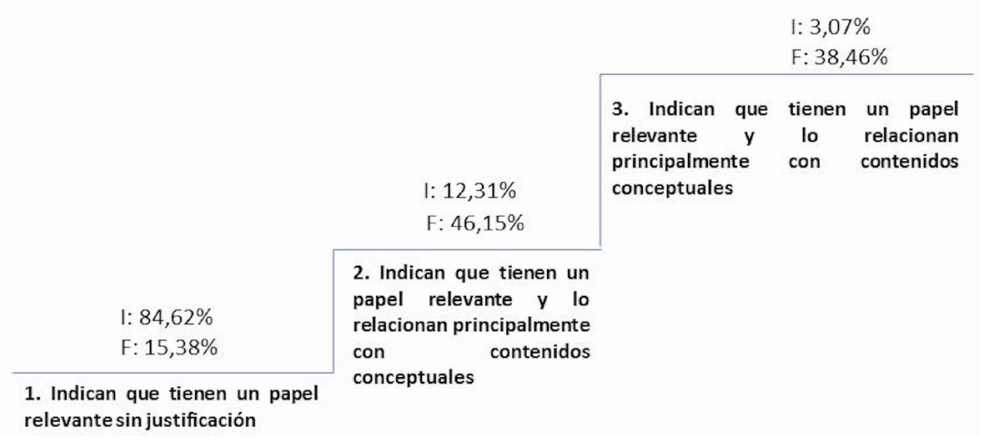

Figura 8. Escalera de aprendizaje Pregunta 6 (primera parte)

Jornadas de Formación e Innovación Docente del Profesorado I № 1 (2018) Esta obra se distribuye con la licencia Creative Commons Reconocimiento-NoComercial-SinObraDerivada Internacional (CC BY-NC-ND 4.0.) 
Finalmente, respecto a la segunda parte de la pregunta 6 , vemos como tanto en el momento inicial como final el nivel 3 es en el que encontramos porcentajes más elevados. Consideramos que esto ocurre en parte porque responden desde un punto de vista ideal, de modo que pensamos que quizás en la puesta en práctica esto sería visto de forma más compleja.

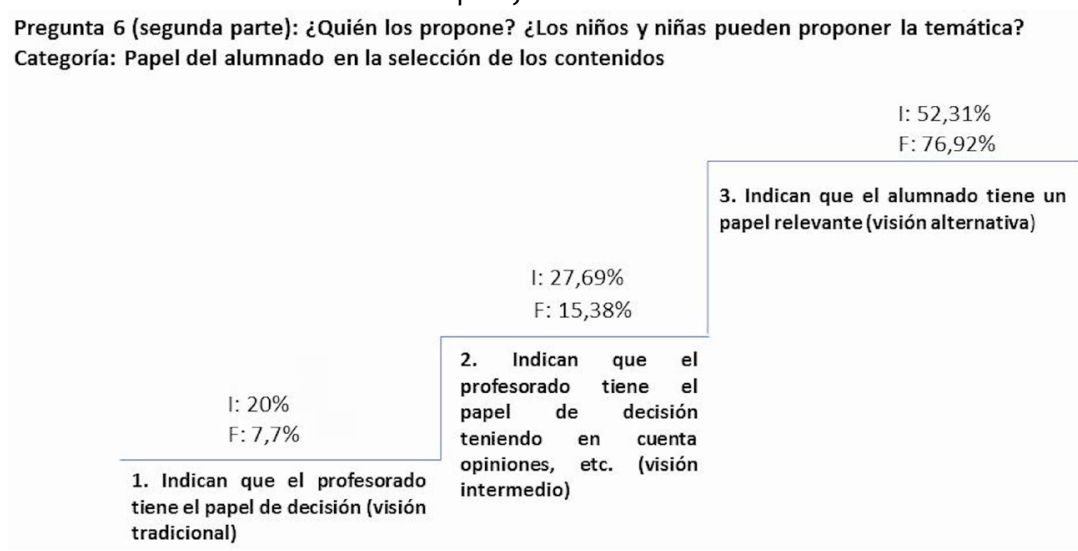

Figura 9. Escalera de aprendizaje Pregunta 6 (segunda parte)

\section{Evaluación del CMD}

Los datos recogidos nos hacen valorar la experiencia de forma satisfactoria, aunque hay que destacar un obstáculo principal que ha llevado a no poder ajustarnos al diseño previo: el tiempo. Realmente, se ha producido un desajuste en la planificación, en cuanto al tiempo asignado a cada uno de los bloques. El hecho de llevar a cabo este CMD de forma paralela a otros bloques y contar solo con las sesiones de los viernes, ha hecho que sea imposible abarcar todos los contenidos previstos. Por otro lado, fuimos conscientes de la necesidad de introducir un bloque de contenidos relacionado con las ideas de los alumnos, que no habíamos considerado en el diseño y creíamos esencial. Esto también hizo que el desajuste en cuanto al tiempo previsto fuera mayor. Por lo tanto, planteamos un nuevo mapa de problemas (Figura 10) y apostamos por 
desarrollar esta unidad completa en mayor tiempo del previsto.

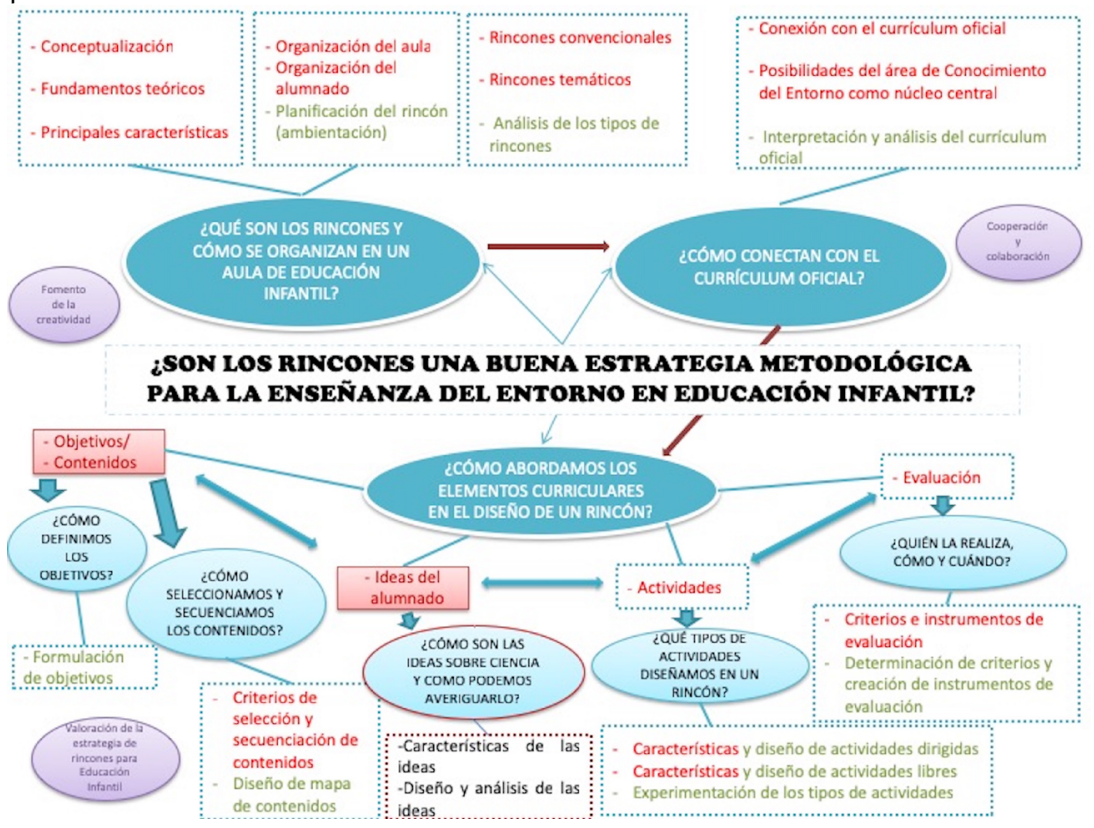

Figura 10. Nuevo mapa de problemas

En cuanto a los aspectos que se pretenden incorporar a la práctica habitual, señalamos la coordinación. De modo que se llevaran a cabo de manera coordinada las dos materias en su totalidad, debiendo para ello de realizar una revisión en profundidad de los bloques de contenidos y diseñando secuencias de actividades conjuntas que permitan al alumnado cursar ambas materias conjuntamente.

Finalmente, en lo que respecta a los principios didácticos que han guiado la experiencia y que deben permanecer en el futuro, señalamos los siguientes. En relación a los contenidos ha primado la necesidad de realizar una buena selección, siempre acorde con los objetivos planteados, siendo para ello fundamental realizar una tarea previa por nuestra parte de análisis epistemológico. Además, hemos considerado esencial trabajar contenidos de distintos tipos: conceptuales, procedimentales y actitudinales, así 
como ser capaces de atribuirles a todos un mismo valor, para evitar que primen unos sobre otros. Un aspecto clave para el desarrollo de esta experiencia ha sido el referente a su organización y relación. Tratar de buscar los nexos que unen los diferentes contenidos, jerarquizarlos y establecer niveles de profundidad, nos ha ayudado bastante a tener más claro qué es lo que verdaderamente queremos que aprendan nuestros alumnos/as y cómo logarlo.

En lo referente a la metodología, de acuerdo a las interesantes aportaciones realizadas por Bain (2007), hemos considerado esencial la creación de un entorno para el aprendizaje crítico natural, en el que el alumnado se comprometa de manera activa en su proceso de aprendizaje. Apostando por el modelo metodológico basado en el principio de investigación (Porlán, 2018).

\section{Referencias bibliográficas}

Bain, K. (2007). Lo que hacen los mejores profesores universitarios. Valencia: Servicio de Publicaciones de la Universidad de Valencia.

Porlán, R. (2018). Enseñanza universitaria. Cómo mejorarla. Madrid: Morata

Jornadas de Formación e Innovación Docente del Profesorado I № 1 (2018) Esta obra se distribuye con la licencia Creative Commons 